\title{
Dysplastic cerebellar gangliocytoma: a description of two cases
}

\author{
Xincui Wan ${ }^{1}$, Hongjuan Chu ${ }^{2}$, Wangsheng Chen ${ }^{3}$, Fei Wang ${ }^{3}$ \\ ${ }^{1}$ Central Sterile Supply Department, Hainan General Hospital, Hainan Affiliated Hospital of Hainan Medical University, Haikou, China; \\ ${ }^{2}$ Department of Pathology, Hainan General Hospital, Hainan Affiliated Hospital of Hainan Medical University, Haikou, China; ${ }^{3}$ Department of \\ Radiology, Hainan General Hospital, Hainan Affiliated Hospital of Hainan Medical University, Haikou, China \\ Correspondence to: Fei Wang. Department of Radiology, Hainan General Hospital, Hainan Affiliated Hospital of Hainan Medical University, \\ 19 Xiu-hua St, Haikou 570311, China. Email: 15103693890@163.com.
}

Submitted May 01, 2020. Accepted for publication Nov 13, 2020.

doi: 10.21037/qims-20-627

View this article at: http://dx.doi.org/10.21037/qims-20-627

\section{Introduction}

Dysplastic cerebellar gangliocytoma (DCG), also known as Lhermitte-Duclos disease (LDD), is a rare, benign, slow-growing, unilateral mass of unclear pathogenesis that occurs in the cerebellum. It is hypothesized to have a malformative, neoplastic, or hamartomatous pathogenesis. On conventional magnetic resonance imaging (MRI), the lesion commonly presents as a mass of striated appearance within the cerebellar hemisphere, with unclear enhancing pattern. In this study, we report two cases of DCG lesions seen on conventional MRI and diffusion-weighted imaging (DWI). To our knowledge, we are first to report the updated enhancement pattern and apparent diffusion coefficient (ADC) information of the lesion seen in DCG.

\section{Case presentations}

A 62-year-old woman presented with a 3-day history of sudden-onset nausea and vomiting, on the background of a 1-year history of unsteady gait. Computed tomography (CT) showed a hypodense left cerebellar mass compared to the unaffected white matter, without obvious calcification. A left cerebellar lesion with hypointensity on T1-weighted image (TR: 1,790 ms; TE: $3 \mathrm{~ms}$ ) was revealed on 3 Tesla MRI (Magnetom Trio, Siemens, Erlangen, Germany). On T2-weighted image (TR: 3,850 ms; TE: $88 \mathrm{~ms}$ ) the mass was hyperintense, with the alternate hyper- and isointensity signals producing a tigroid appearance (Figure 1). The lesion showed a hyperintense signal on T2-fluid-attenuated inversion recovery (FLAIR) (TR: 7,000 ms; TE:118 ms).
On the ADC image, 10 manually drawn circular regions of interest (ROIs) were created in the DCG mass. A comparison was made with a comparably positioned ROI in the normal contralateral cerebellum, which showed elevated ADC in the mass compared with the normal cerebellum $\left[(863.1 \pm 78.0)\right.$ and $(660.4 \pm 56.5) \times 10^{-6} \mathrm{~mm}^{2} / \mathrm{s}$ respectively; $\mathrm{P}<0.001$ ] (Table 1). Volumetric sagittal and conventional axial T1-weighted images with contrast enhancement were available, which showed thin, hairy blood vessels within and along the periphery of the lesion. Compression of the unaffected right side during operation was found because of expansion of the left side of the cerebellum. The tumor was pale in color, soft in consistency, and moderately vascular, originating from the cerebellar tissue and displaying an illdefined margin. Histopathological examination revealed a relatively preserved cerebellar architecture with variably sized dysplastic ganglion cells. Ectatic vessels were observed, with neither mitosis nor necrosis.

A 66-year-old woman presented with a 2-year history of gradually worsening dizziness, on a background of 8 years of intermittent dizziness and thyroid nodule surgery several years ago. CT showed a hypodense mass in the right cerebellar hemisphere without skull remodeling, while MRI revealed a lobulated right cerebellar lesion with tigroid appearance (Figure 2). The signal intensity of the lesion was higher on T2-FLAIR and DWI, and hemosiderin deposition was revealed along the periphery of the lesion on a T2-weighted image. Based on a paired analysis, the ADC was universally elevated in DCG compared with the contralateral cerebellum $\left[(931.3 \pm 101.1) \times 10^{-6} \mathrm{~mm}^{2} / \mathrm{s}\right.$ and $(724.3 \pm 56.5) \times 10^{-6} \mathrm{~mm}^{2} / \mathrm{s}$ respectively; $\mathrm{P}<0.0001$ ] (Table 1). 

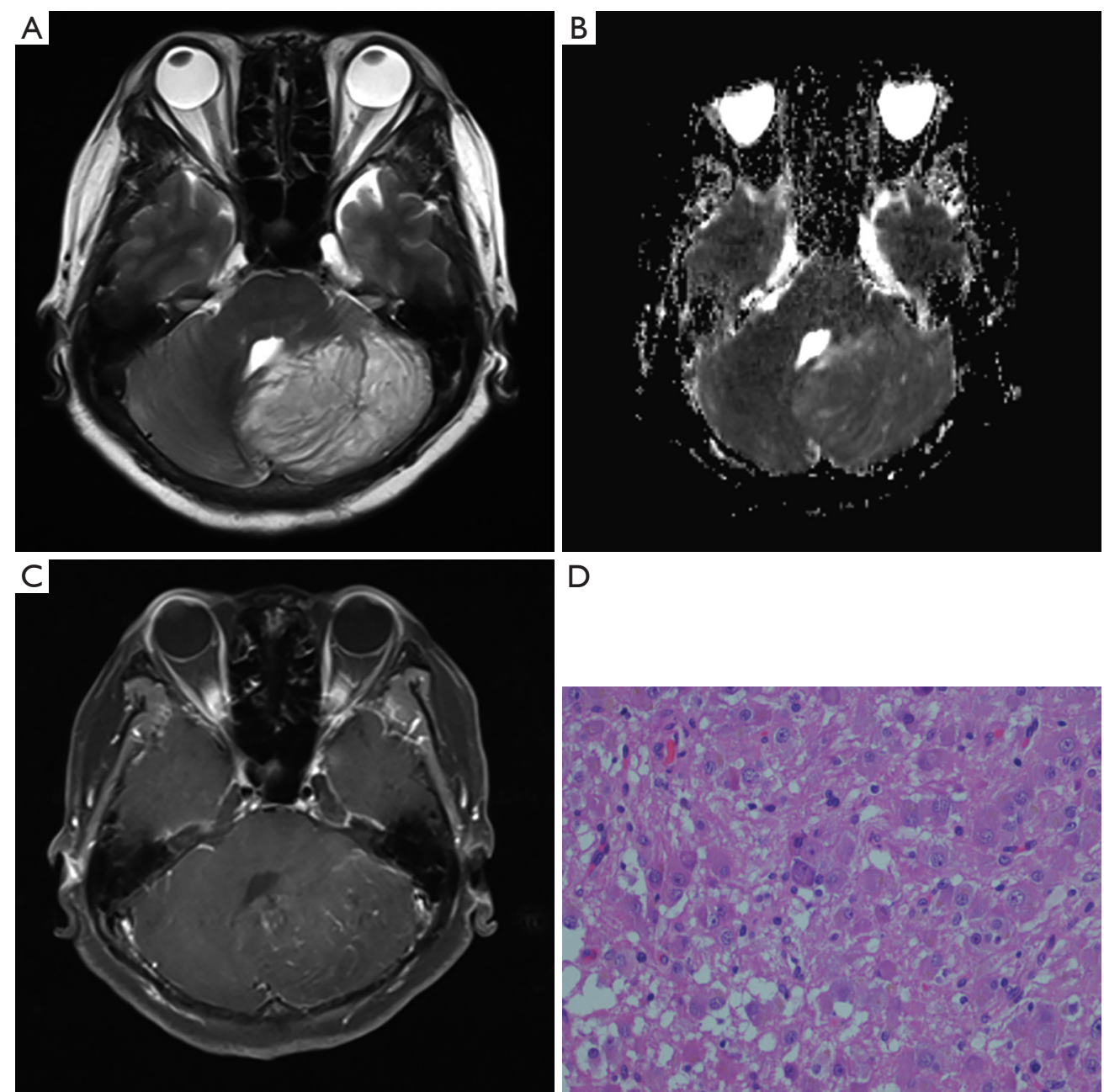

D

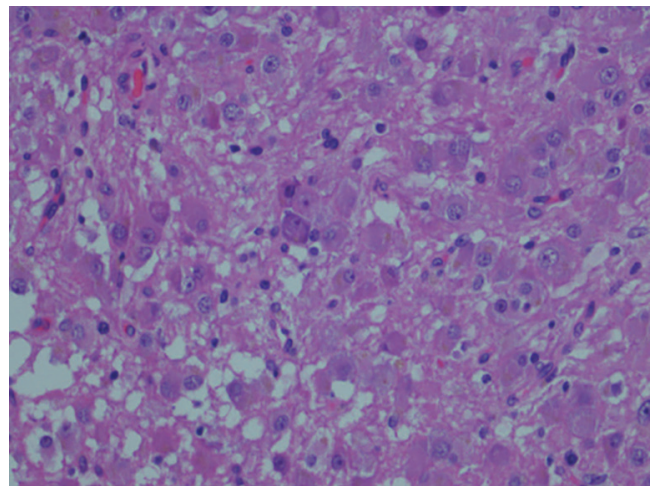

Figure 1 Left cerebellar hemisphere. (A) Axial T2-weighted image reveals a heterogeneously high-signal mass whose laminated architecture is characteristic of DCG. (B) ADC map shows increase in diffusion. (C) T1-weighted image with contrast enhancement reveals numerous veins running between the folia. (D) Photomicrograph (HE staining, 400× original magnification) reveals numerous dysplastic ganglion cells, rich cytoplasm, nuclear offset, and vacuolated clear nucleoli. DCG, Dysplastic cerebellar gangliocytoma; ADC, apparent diffusion coefficient.

A T1-weighted image with contrast enhancement demonstrated small curvilinear enhancing vessels within or along the periphery of the lesion with associated vermis involvement. With non-surgical management and routine follow-up for 3 years, the mass remained stable.

\section{Discussion}

Lhermitte and Duclos first reported DCG in 1920, and since this discovery, more than 200 cases have been reported globally. The 2016 revised fourth edition of the World Health Organization (WHO) Classification of Tumours of the Central Nervous System stated that DCG lesions typically do not enhance (1). To our knowledge, we are first to report an updated enhancement pattern. The DCG lesions presented no enhancement, with thin hairy blood vessels seen within or along the periphery of the lesion with an elevated ADC mass.

DCG usually presents in the third and fourth decades of life and rarely in children (2-4), with no gender or race preponderance. Size of lesion, laterality, and disease duration vary considerably (5). Most patients have clinical symptoms related to gradual or sudden increased intracranial pressure and obstructive hydrocephalus; 
Table 1 ADC features of DCG

\begin{tabular}{|c|c|c|c|c|}
\hline Case & Affected & Unaffected & $\mathrm{t}$ & $P$ \\
\hline \multirow[t]{10}{*}{1} & 831.1 & 568.6 & 7.637 & $<0.001$ \\
\hline & 815.0 & 605.5 & & \\
\hline & 923.8 & 837.7 & & \\
\hline & 859.3 & 599.6 & & \\
\hline & 800.8 & 706.2 & & \\
\hline & 835.3 & 618.6 & & \\
\hline & $1,037.8$ & 693.9 & & \\
\hline & 826.7 & 560.5 & & \\
\hline & 776.5 & 624.3 & & \\
\hline & 924.4 & 789.0 & & \\
\hline \multirow[t]{10}{*}{2} & $1,015.4$ & 701.4 & 7.352 & $<0.001$ \\
\hline & 856.9 & 734.0 & & \\
\hline & 881.9 & 699.0 & & \\
\hline & $1,135.9$ & 860.6 & & \\
\hline & 813.7 & 746.2 & & \\
\hline & 859.7 & 695.7 & & \\
\hline & $1,003.1$ & 688.9 & & \\
\hline & 839.0 & 680.8 & & \\
\hline & 980.2 & 671.5 & & \\
\hline & 926.9 & 764.9 & & \\
\hline
\end{tabular}

ADC, apparent diffusion coefficient; DCG, Dysplastic cerebellar gangliocytoma.

however, some may be asymptomatic.

DCG has unique histopathological characteristics. Histopathological examination typically reveals hypertrophic ganglion cells expanding the granular and molecular layers of the cerebellar cortex and abnormally increased myelination in the molecular layer. A marked reduction in myelination of the central white mater of the cerebellar folia is also common. Absence of mitotic activity, necrosis, and endothelial proliferation are characteristic in these lesions, while malignant transformation is uncommon (6).

On non-enhanced CT, the DCG is usually hypodense. While isodense, secondary abnormalities—such as deformation of the fourth ventricle or pons, effacement of the cerebellopontine angle cistern, obstructive hydrocephalus, cerebellar tonsil hernia, and potential calcifications-may help guide diagnosis, CT remains unfavorable because of the beam-hardening artifacts caused by the petrous temporal bone which impede visualization.

MRI is thus the imaging modality of choice. The central core of T1-hypointensity and T2-hyperintensity corresponds with the thinned white matter, widened granular cell layer, and inner portions of the dysplastic molecular layer. The outer thinner layer (T1-isointense and T2-iso- to hypointense) can be attributed to the outer molecular layer and the leptomeninges, associated with abnormal vessels and foci of calcification (7). A lack of contrast enhancement has been regarded as an important MRI diagnostic criterion for DCG, which suggests no significant blood-brain barrier damage and no extracellular edema (8). In our patient, the veins surrounding the thickened folia were well-demonstrated by the T1-weighted image with contrast enhancement and well-correlated with the pathological feature of the ectatic vessels. Some researchers may mistake venous proliferation as contrast enhancement, as venous proliferation may present flow voids on MRI (9). Previous reports indicate perfusion-weighted imaging increased perfusion compared to adjacent cerebellar tissue, and hyperperfusion without enhancement was found to correlate closely to the histopathologic observation of numerous dilated thin-walled blood vessels (10).

Consistent with general consensus, DWI in our cases showed a hyperintense area in the lesion due to the "T2 shine-through". In the ADC map, the mass appeared isointense relative to the unaffected cerebellar parenchyma; however, contrary to previous reports, the masses in our cases showed elevated ADC. The signals on DWI and ADC maps depend on cell density, low extracellular water content, and enhancement of the tumor (10); hence, the present findings on DWI might have resulted from dysplastic cortical neurons in the granular layer, a thickening of the molecular layer, loss of Purkinje cells, and a thinning of white matter. MRI findings in our case are in accordance with other published cases (5).

The limitation of this study is the lack of other magnetic resonance methods, including susceptibility-weighted imaging, perfusion-weighted imaging, and magnetic resonance spectroscopy, which precludes correlation analysis and immunohistochemical analysis of results.

\section{Conclusions}

The DCG lesion is a rare, benign, cerebellar mass that can be diagnosed from its unique magnetic resonance features. Although the exact pathophysiological mechanism remains 

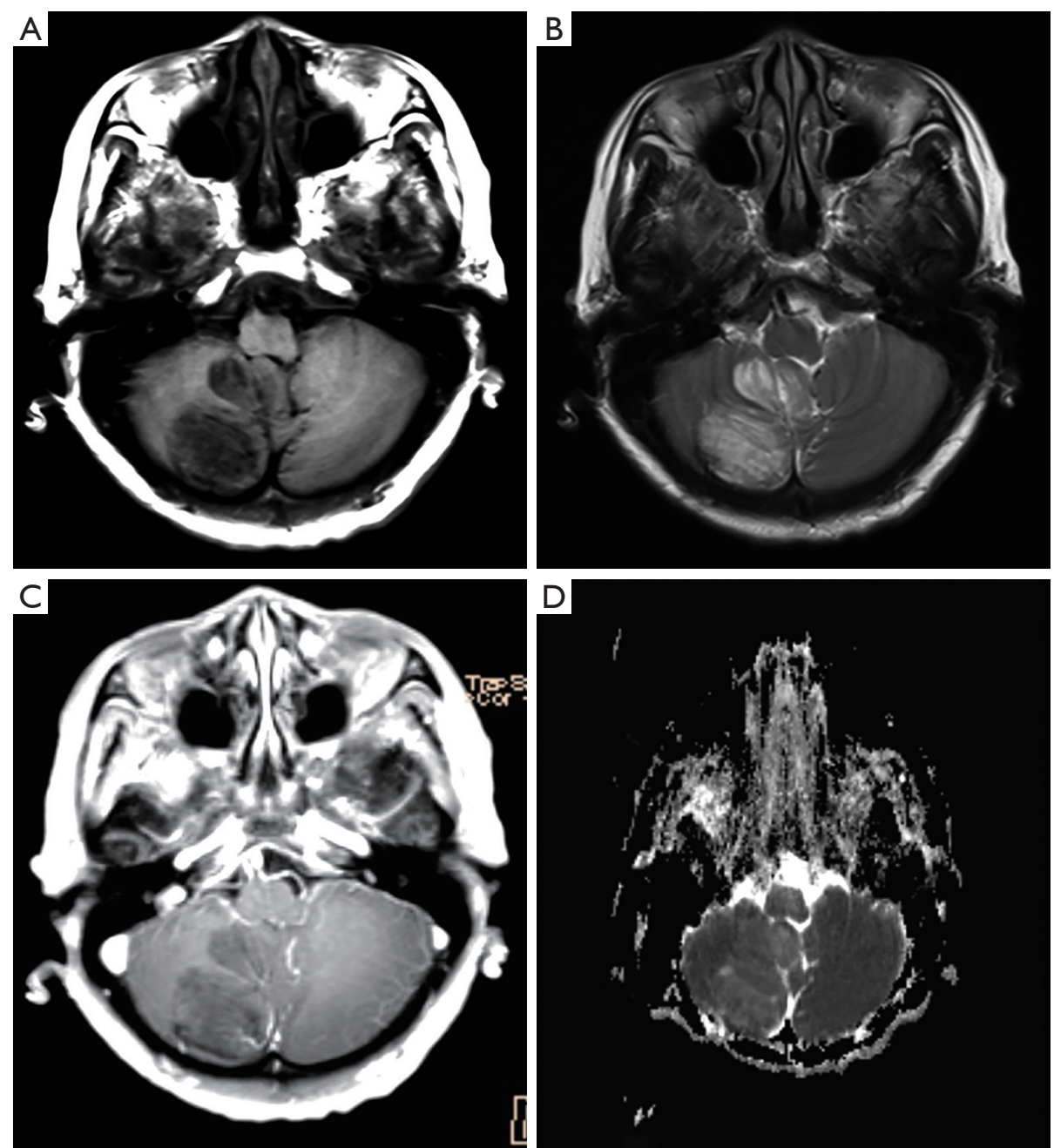

Figure 2 Right cerebellar hemisphere. Axial T1-weight image (A) and axial T2-weighted image (B) reveal a striated-pattern mass in accordance with DCG. (C) A contrast-enhanced axial T1-weighted image shows veins running along the lesion. (D) The ADC map shows an increase in diffusion. ADC, apparent diffusion coefficient.

unclear, features of tigroid appearance; a lack contrast enhancement; thin, hairy blood vessels within or along the periphery of the lesion; and an elevated ADC mass, are sufficient to make a correct diagnosis.

\section{Acknowledgments}

Funding: This study was supported by the National Natural Science Foundation (no. 81360217) and the Hainan Province Science Technology Project (no. ZDYF2016108).

\section{Footnote}

Conflicts of Interest: All authors have completed the ICMJE uniform disclosure form (available at http://dx.doi. org/10.21037/qims-20-627). The authors have no conflicts of interest to declare.

Ethical Statement: The authors are accountable for all aspects of the work in ensuring that questions related to the accuracy or integrity of any part of the work are appropriately investigated and resolved. All procedures performed in studies involving human participants were in accordance with the ethical standards of the institutional and/or national research committee(s) and with the Helsinki Declaration (as revised in 2013). Written informed consent was obtained from the patients for publication of this study and any accompanying images. 
Open Access Statement: This is an Open Access article distributed in accordance with the Creative Commons Attribution-NonCommercial-NoDerivs 4.0 International License (CC BY-NC-ND 4.0), which permits the noncommercial replication and distribution of the article with the strict proviso that no changes or edits are made and the original work is properly cited (including links to both the formal publication through the relevant DOI and the license). See: https://creativecommons.org/licenses/by-nc-nd/4.0/.

\section{References}

1. Louis DN, Ohgaki H, Wiestler OD, Cavenee WK. World Health Organization Histological Classification of Tumours of the Central Nervous System. International Agency for Research on Cancer, France. 2016.

2. Golden N, Tjokorda MG, Sri M, Niryana W, Herman S. Management of unusual dysplastic gangliocytoma of the cerebellum (Lhermitte-Duclos disease) in a developing country: Case report and review of the literature. Asian J Neurosurg 2016;11:170.

3. Ezgu MC, Ozer MI, Dogan A, Deveci G, Kural C, Izci Y. Lhermitte-Duclos Disease in a Six-Year Old Child: A Rare Presentation. Pediatr Neurosurg 2018; 53:416-20.

4. Abi Lahoud G, Chalouhi N, Zanaty M, Rizk T, Jabbour P. Association of Lhermitte-Duclos disease and split cord malformation in a child. J Clin Neurosci 2014;21:1999-2002.
5. Dhamija R, Wood CP, Porter AB, Hu LS, Weindling SM, Hoxworth JM. Updated Imaging Features of Dysplastic Cerebellar Gangliocytoma. J Comput Assist Tomogr 2019;43:277-81.

6. Hariri OR, Khachekian A, Muilli D, Amin J, Minassian T, Berman B, Ritter Y, Siddiqi J. Acute-onset cerebellar symptoms in Lhermitte-Duclos disease: Case report. Cerebellum 2013;12:127-30.

7. Cheng CS, Ou CH, Chen JS, Lui CC, Yeh LR. LhermitteDuclos disease: A case report with radiologic-pathologic correlation. Radiol Case Rep 2019;14:734-9.

8. Moonis G, Ibrahim M, Melhem ER. Diffusion-weighted MRI in Lhermitte-Duclos disease: report of two cases. Neuroradiology 2004;46:351.

9. Spaargaren L, Cras P, Bomhof MA, Lie ST, de Barsy AM, Croese PH, Teepen JL, Duwel VH, Van Goethem JW, Ozsarlak O, van den Hauwe L, De Schepper AM, Parizel PM. Contrast enhancement in LhermitteDuclos disease of the cerebellum: correlation of imaging with neuropathology in two cases. Neuroradiology 2003;45:381-5.

10. Klisch J, Juengling F, Spreer J, Koch D, Thiel T, Büchert M, Arnold S, Feuerhake F, Schumacher M. LhermitteDuclos disease: assessment with MR imaging, positron emission tomography, single-photon emission CT, and MR spectroscopy. Am J Neuroradiol 2001;22:824-30.
Cite this article as: Wan $\mathrm{X}$, Chu $\mathrm{H}$, Chen W, Wang F. Dysplastic cerebellar gangliocytoma: a description of two cases. Quant Imaging Med Surg 2021;11(11):4695-4699. doi: 10.21037/ qims-20-627 